\title{
ERROS NA ADMINISTRAÇÃO DE MEDICAMENTOS: DIVULGAÇÃO DE CONHECIMENTOS E IDENTIFICAÇÃO DO PACIENTE COMO ASPECTOS RELEVANTES
}

\author{
ERROS IN THE ADMINISTRATION OF MEDICATIONS: THE SCIENTIFIC \\ KNOWLEDGE AND THE IDENTIFICATION OF THE PATIENT \\ AS AN IMPORTANT ASPECTS
}

Adriana Inocenti Miasso* Silvia Helena De Bortoli Cassiani**

MIASSO, A. I.; CASSINI, S. H. DE B. Erros na adiministração de medicamentos: divulgação de conhecimentos e identificação do paciente como aspectos relevantes. Rev.Esc.Enf.USP, v. 34, n. 1, p. 16-25, mar. 2000.

\section{RESUMO}

A administração de medicamentos é um processo multidisciplinar e um multi-sistema. Erros podem ocorrer em todas as etapas desse processo. Estratégias podem ser utilizadas e devem ser divulgadas para minimizar a ocorrência de erros. Este estudo foi planejado tendo os seguintes objetivos: identificar e analisar em periódicos das áreas de enfermagem, farmácia e hospitalar, publicações com temas ligados a administração de medicamentos e verificar a identificação no leito e no antebraço de pacientes internados em uma instituição hospitalar. Para alcançar o primeiro objetivo realizou-se uma revisão da literatura sobre a temática em estudo, em três periódicos de maior circulação nacional das áreas descritas, no periodo de 1987 a 1997. Os artigos identificados foram agrupados em 5 tipos: assistência de enfermagem, ensino, medicamentos, pesquisa e técnica. Identificou-se 19 artigos sobre a temática estudada, sendo 42,1\% destes voltados para os aspectos técnicos da administração de medicamentos. Para atingir o segundo objetivo realizou-se uma investigação de um dia, escolhido casualmente, verificando-se os 360 leitos da instituição quanto a identificação nos mesmos e nos pacientes. Destes, $74,7 \%$ estavam ocupados e 75,8\% devidamente identificados. Observou-se que 23,8\% dos pacientes possuiam pulseira de identificação colocada, na maioria dos pacientes, no dia anterior à cirurgia e não no momento da internação. Os resultados evidenciaram a pouca importância tanto do estudo desta temática, quanto na identificação do paciente e do leito por parte da instituição. Há pois necessidade de aumentar a divulgação de estudos e incrementar ações que minimizema ocorrência de erros na medicação.

PALAVRAS-CHAVE: Medicamentos. Erros de medicação.Pacientes internados.

The administration of medications is a multi-system process. Errors can happen in all the stages of that process. Strategies can be used and it should be published to minimize the occurrence of errors. This study had the following objectives: to identify and to analyze publications with themes about the administration of medication in nursing, hospital and pharmacy journals and to verify the identification in the bed and in the patients'forearm in impatient clinics. To reach the first objective it was realized a revision of the literature in three journals of larger national circulation of the described areas, in the period from 1987 to 1997. The identified articles were classified in 5 types: nursing staff, teaching, medications, research and technique. It was identified 19 articles on the thematic studied, being 42,1\% of these related to the technical aspects of the administration of medications. To reach the second objective it was taken an investigation of one day, chosen accidentally, being verified the identification of the beds and the patients in the 360 beds of the institution. Of these, 74,7\% were busy and 75,8\% properly identified. It was observed that $23,8 \%$ of the patients have gotten bracelet of placed identification, in the day previous to the surgery and not in the moment of the hospitalization. The results evidenced the need to increase the publication of the studies about errors and to increase actions that minimize the occurrence of errors in the medication in impatient patients.

KEYWORDS: Medications. Medications errors. Inpatients.

\footnotetext{
* Enfermeira. Bolsista de Aperfeiçoamento do CNPq.

** Professora Doutora do Departamento de Enfermagem Geral e Especializada da EERP-USP. Coordenadora do Projeto Integrado: "O processo tia administração de medicamentos: fatores de risco e avanços tecnológicos, ciêntíficos e educacionais."
} 


\section{INTRODUÇÃO}

Nas instituições hospitalares é responsabilidade dos profissionais de enfermagem assegurar aos pacientes uma assistência livre de imprudência, impericia ou negligência. Entretanto, erros relacionados à administração de medicamentos ocorrem em muitos hospitais podendo trazer sérias conseqüências para os pacientes, instituição e profissionais.

Segundo a American Society of Health-System Pharmacists - ASHP (1998) os erros de medicação em hospitais americanos afetam entre 2 a 14\% dos pacientes admitidos. Embora grande número destes erros não causem complicações, alguns podem tornar-se sérios $0^{\bullet}$ suficiente causando danos ou o óbito.

PEPPER (1995) define um erro na administração de medicamento como uma discrepância entre a terapia medicamentosa recebida pelo paciente e a terapia planejada pelo médico. BOOTH (1994) classifica os erros em cinco tipos: erros relativos à prescrição e estocagem dos medicamentos, à informação/comunicação, ao paciente, à administração e aos aspectos gerais envolvidos na ocorrência de erros ao ministrar um medicamento. No que se refere aos erros relativos ao paciente, são incluídas por este autor, as falhas ao identificar o mesmo.

Para administrar um medicamento com segurança e eficiência, o enfermeiro deve conhecer a ação do mesmo no organismo, métodos e vias de administração e eliminação, reações colaterais, dose máxima e terapêutica, efeitos tóxicos, além do conhecimento da técnica de administração e do cliente. Estes requisitos não estão presentes na grande maioria das instituições brasileiras, onde a responsabilidade da administração de medicamentos está nas mãos de auxiliares e técnicos de Enfermagem, atuando muitas vezes sem supervisão de enfermeiros, em organizações que nem sempre priorizam a qualidade do cuidado.

O enfermeiro, embora não sendo o responsável pela prescrição do medicamento, deve conhecer todos os aspectos e fases envolvidas na administração do mesmo, a fim de prevenir erros e enganos que lesem o paciente.

O sistema de distribuição de medicamentos aos pacientes envolve vários profissionais como: médicos, farmacêuticos, enfermeiros, ocupacionais de enfermagem e, em algumas instituições, há a participação do escriturário; no entanto, a equipe de enfermagem constitui o elo final deste sistema atuando na administração propriamente dita e por este motivo, é geralmente a responsável pelos atos que marcam a transição de um erro prevenivel para um erro real e, o ônus dos erros, cai pesadamente sobre este profissionais.

Pode-se afirmar que a administração de medicamentos constitui um processo multidisciplinar e um multi-sistema, iniciando no momento da prescrição médica, continuando com a provisão deste medicamento pelo farmacêutico e terminando com a sua preparação e administração aos clientes. Erros ou intercorrências podem ocorrer em qualquer uma destas etapas (CASSIANI; BUENO, 1998).

Para garantir a segurança na administração de medicamentos, os cinco certos devem ser observados: medicamento certo, paciente certo, dose certa, via de administração certa e horário certo. Destes itens, o "paciente certo", será sempre o desafio para os profissionais se estes não utilizarem estratégias necessárias para assegurar que realmente o paciente receba sua medicação prescrita. Destas estratégias, destacam-se a identificação dos pacientes através de pulseiras contendo os nomes destes em letras legiveis, o questionamento do paciente de seu nome completo, a identificação do leito e evitar que pacientes com nomes semelhantes ocupem a mesma enfermaria.

PEPPER (1995) apresenta dados de um estudo onde notou-se que mais de $11,4 \%$ dos pacientes de uma instituição estudada não tinham a pulseira de identificação, e esta havia sido removida para facilitar o acesso venoso ou para o conforto do paciente. Para MIQUELIN; CASSIANI; BUENO(1998), quando a medicação de um paciente é administrada em outro, ocorrem dois erros: o erro de omissão, pois o paciente que deveria receber a medicação não a recebeu, e um erro classificado como administração de um medicamento não autorizado para o paciente que a recebeu e não deveria receber.

COBB (1986) afirma que a pulseira de identificação do paciente deve também conter a alergia a determinado medicamento; dessa forma, se a Enfermagem administra no paciente um medicamento ao qual o mesmo é alérgico, a verificação do medicamento no registro e no braço do paciente deve ter sido negligenciada, ocasionando um erro de séria natureza.

Estudo conduzido por MIQUELIN; CASSIANI; BUENO (1998) identificou que o erro mais freqüente foi a medicação administrada a paciente errado. Diante do exposto e sabendo que a identificação do leito e a identificação no próprio paciente através de "pulseira de identificação" devem ser usadas como formas de evitar a ocorrência de erros em pacientes, conduzimos este estudo buscando identificar a presença destes elementos nos pacientes de uma referida instituição. 


\section{OBJETIVOS}

Esta investigação teve como objetivos:

- Identificar e analisar publicações em periódicos de maior circulação nacional nas áreas de Enfermagem, farmácia e hospitalar, que abordem temas ligados à administração de medicamentos, especificamente erros .

- Verificar a presença de identificação adequada no leito e no antebraço de pacientes internados em uma Instituição Hospitalar do interior de São Paulo.

\section{MATERIAIS E MÉTODOS}

A trajetória percorrida para a elaboração deste estudo pode ser didaticamente dividida em dois momentos. Inicialmente, foi realizada uma revisão da literatura sobre a temática nos três periódicos de maior circulação nacional nos últimos dez anos (1987 a 1997), nas áreas de enfermagem, farmácia e hospitalar.

Os locais utilizados para revisão foram: Biblioteca Central do Campus de Ribeirão Preto USP, Sala de Leitura Glete de Alcântara - EERP USP, arquivos LILACS e MEDLINE. Na área de Enfermagem, os periódicos analisados foram: Revista Gaúcha de Enfermagem, Revista Brasileira de Enfermagem e Revista Latino Americana de Enfermagem. Em farmácia foram revisadas a Revista Brasileira de Farmácia, a Revista de Farmácia e Bioquímica da USP e a Revista do Farmacêutico. Já na área hospitalar, procedeu-se a análise da Revista do Hospital das Clinicas da F.M.U.S.P e Revista Paulista de Hospitais, pois nesta área foram encontrados apenas dois periódicos contendo todos os números referentes aos anos em estudo.

Os artigos identificados foram agrupados em 5 tipos: assistência de enfermagem, ensino, medicamento, pesquisa e técnica e a seguir analisados.

Posteriormente, realizou-se uma investigação de um dia, escolhido casualmente, onde foram verificados os 360 leitos de um hospital de ensino do interior do Estado de São Paulo, verificando a presença de identificação nos mesmos e no antebraço dos pacientes hospitalizados.

Elaborou-se um instrumento em que se questionava o paciente se ele conhecia a pulseira de identificação, se já havia feito uso nessa internação, se foi tirada e o porquê.

\section{ANÁLISE E DISCUSSÃO}

\section{LEVANTAMENTO BIBLIOGRÁFICO}

$\mathrm{Na}$ literatura internacional, a maioria dos estudos sobre erros na administração de medicamentos têm sido desenvolvidos por farmacêuticos. Apesar do crescimento fenomenal das pesquisas em Enfermagem e do fato de que a administração de medicamentos pertence legalmente e tradicionalmente à prática de Enfermagem, verifica-se uma carência de investigadores sobre o assunto (PEPPER, 1995).

Dessa forma, interessadas em investigar a ocorrência de publicações que abordem temas direta ou indiretamente ligados à erros na administração de medicamentos, realizamos um levantamento bibliográfico dos três periódicos nacionais de maior circulação nas áreas de Enfermagem, farmácia e hospital e cujos dados coletados apresentamos no Quadro 1. 
QUADRO 1 - Distribuição de artigos nacionais analisados segundo revista, ano, volume, número, autor e título do trabalho na área de Enfermagem, no período de 1987 a 1997.

\begin{tabular}{|c|c|c|c|}
\hline REVISTA & ANO & VOLUME E $\mathbf{N}^{\circ}$ & AUTOR E TÍTULO DO TRABALHO \\
\hline $\begin{array}{l}\text { Revista Gaúcha } \\
\text { de Enfermagem }\end{array}$ & 1987 & $\mathrm{~V} .8 \mathrm{~N}^{\circ} 2$ & $\begin{array}{c}\text { Duarte, M;N.N.; et al. Assistência de } \\
\text { enfermagem na aplicação de sulfato de } \\
\text { magnésio em pacientes portadoras de pré- } \\
\text { esclâmpsia grave e esclâmpsia no H.C. de Porto } \\
\text { Alegre. } \\
\text { MILANZES, H.M.; ALMERÃO, E. O papel da } \\
\text { enfermeira na orientação e administraç ao de } \\
\text { drogas que levam à imunossupressão em } \\
\text { pacientes submetidos à Transplante Cardíaco. }\end{array}$ \\
\hline \multirow[t]{4}{*}{$\begin{array}{l}\text { Revista } \\
\text { Brasileira de } \\
\text { Enfermagem }\end{array}$} & 1988 & $\mathrm{~V} .41 \mathrm{~N}^{\circ} 2$ & $\begin{array}{l}\text { MENDES, I. A. C.; et al.. A administração de } \\
\text { injetáveis em farmácias: ação educativas do } \\
\text { enfermeiro. }\end{array}$ \\
\hline & 1991 & $\begin{array}{l}\mathrm{V} .44 \mathrm{~N}^{\circ} 2 \\
\mathrm{~V} .44 \mathrm{~N}^{\circ} 3\end{array}$ & $\begin{array}{c}\text { DUARTE, M.J.R.S. Influência da indústria } \\
\text { farmacêutica na politica de medicamentos, } \\
\text { segundo os profissionais de saúde. } \\
\text { MAEDA, S.T.; CAMPEDELLI, M.C. Escalpe e } \\
\text { equipo de soro: opinião de enfermeiras quanto } \\
\text { aos requisitos essenciais de qualidade. } \\
\text { NASCIMENTO, E.M.F.; CANÇADO, M.L.B. O } \\
\text { uso de um sistema artesanalmente construido } \\
\text { para o ensino prático da punção e infusão } \\
\text { intravenosa. }\end{array}$ \\
\hline & 1992 & V.45 $\mathrm{N}^{\circ} 4$ & $\begin{array}{l}\text { MIYADAHIRA, A.M.K.; KOIZUMI, M.S. Medida } \\
\text { das capacidades psicomotoras envolvidas na } \\
\text { técnica da injeção intramuscular. }\end{array}$ \\
\hline & 1994 & $\mathrm{~V} .47 \mathrm{~N}^{\circ} 3$ & $\begin{array}{c}\text { BARALDI, S.; OLIVEIRA, S.L.; OLIVEIRA, M.A. } \\
\text { A utilização da região ventro-glútea para } \\
\text { aplicação da vacina dupla-adulto: opinião da } \\
\text { clientela. }\end{array}$ \\
\hline $\begin{array}{l}\text { Revista Latino- } \\
\text { Americana de } \\
\text { Enfermagem }\end{array}$ & 1993 & V.1 N Especial & $\begin{array}{l}\text { FÁTIMA, A.P. A temática administração de } \\
\text { medicamentos nas pesquisas em enfermagem }\end{array}$ \\
\hline
\end{tabular}

Observando o quadro 1 , percebemos que na área de Enfermagem as publicações sobre esta temática encontram-se durante o período de 1987 a 1994 perfazendo um total de nove artigos. Fica evidente um maior número de publicações pela
Revista Brasileira de Enfermagem com um total de seis publicações no período em estudo.

No que concerne às revistas da área hospitalar, o Quadro 2 apresenta os artigos investigados. 
QUADRO 2 - Distribuição de artigos nacionais analisados segundo revista, ano, volume, número, autor e título do trabalho na área de hospital, no período de 1987 a 1997.

\begin{tabular}{|c|c|c|c|}
\hline REVISTA & ANO & VOLUME E $\mathbf{N}^{\circ}$ & AUTOR E TÍTULO DO TRABALHO \\
\hline \multirow[t]{4}{*}{$\begin{array}{l}\text { Revista } \\
\text { Paulista de } \\
\text { Hospitais }\end{array}$} & 1987 & $4 / 5 / 6$ & $\begin{array}{l}\text { ROSA, V. H. C. T.; PIVELLO, V.L. Antibióticos } \\
\text { e antineoplásicos de uso injetável: cuidados } \\
\text { nos procedimentos de manuseio: fator de } \\
\text { segurança e economia. }\end{array}$ \\
\hline & & $7 / 8 / 9$ & $\begin{array}{l}\text { BRAZZACH, M.L.; BERTUZZI, H.J.; } \\
\text { BOLYHOS, I. Cuidados na administração de } \\
\text { alguns medicamentos injetáveis. }\end{array}$ \\
\hline & 1988 & $1 / 2 / 3$ & $\begin{array}{c}\text { SCAIN, S.F. Reutilização de seringa } \\
\text { descartável para aplicação de insulina. }\end{array}$ \\
\hline & & $10 / 11 / 12$ & $\begin{array}{l}\text { CZAPSKI, J.; et al. Normas para o manuseio } \\
\text { em serviços de saúde de soluções de grande } \\
\text { volume para uso parenteral. }\end{array}$ \\
\hline
\end{tabular}

Observa-se através do quadro 2 que dos periódicos revisados na área de hospital, apenas a Revista Paulista de Hospitais apresentou artigos sobre a temática estudada. Foram encontrados quatro trabalhos estando estes distribuídos uniformemente nos dois primeiros anos em estudo(1987 e 1988). Em relação aos autores, apresentam o mesmo número de publicações no período estudado.

Quanto às revistas da área de farmácia, foram identificados os seguintes artigos apresentados no Quadro 3.

QUADRO 3 - Distribuição de artigos nacionais analisados segundo revista, ano, volume, número, autor e título do trabalho na área de farmácia, no período de 1987 a 1997.

\begin{tabular}{|c|c|c|c|}
\hline REVISTA & ANO & VOLUME E N ${ }^{\circ}$ & AUTOR E TÍTULO DO TRABALHO \\
\hline $\begin{array}{l}\text { Revista } \\
\text { Brasileira de } \\
\text { Farmácia }\end{array}$ & 1991 & V.72 $\mathrm{N}^{\circ} 1$ & $\begin{array}{l}\text { SANTORO, M.I.R.M. Dissolução de } \\
\text { medicamentos no Brasil: urgência na } \\
\text { padronização. }\end{array}$ \\
\hline & 1992 & V.73 $\mathrm{N}^{\circ} 4$ & $\begin{array}{c}\text { ROSA, V.H.C.T.; MARIN, M.L.M.; BARBOSA, } \\
\text { A.O. Requisitos necessários para o preparo } \\
\text { asséptico das soluções de Nutrição Parenteral } \\
\text { Prolongada (NPP). } \\
\text { LISBOA, S.M.; GRABE, A. O emprego de } \\
\text { colheres domésticas para medir xaropes e } \\
\text { suspensões medicamentosas. }\end{array}$ \\
\hline & 1994 & V.75 $\mathrm{N}^{\circ} 4$ & $\begin{array}{c}\text { CASTRO, C.G.S.O.; RIBEIRO, D.C.S.; PITTA, } \\
\text { A.C.M. Perfil de prescrição de soluções } \\
\text { nutritivas parenterais no IFF/FIO CUZ: } \\
\text { estudo em três enfermarias pediátricas, } \\
1993 / 94 .\end{array}$ \\
\hline & 1996 & V. $77 \mathrm{~N}^{\circ} 3$ & $\begin{array}{l}\text { CASTRO, C.G.S.O.; RIBEIRO, D.C.S. Protocolo } \\
\text { para aquisição de medicamentos em } \\
\text { instituições públicas de saúde. } \\
\text { CARESTIATO, J.C.; PEREIRA, L.G. Dose } \\
\text { unitária: relação custo x beneficio de sua } \\
\text { implantação nos hospitais públicos brasileiros } \\
\text { como política de governo. }\end{array}$ \\
\hline
\end{tabular}


Dos três periódicos pesquisados na área de farmácia (Revista Brasileira de Farmácia, Revista de Farmácia e Bioquímica da USP e Revista do Farmacêutico), foram encontrados um total de seis artigos, todos publicados na Revista Brasileira de Farmácia (Quadro 3). As publicações encontram-se durante o período de 1991 a 1996 com um maior número de publicações no segundo semestre de cada ano. Com relação aos autores, verifica-se que duas publicações possuem os mesmos autores.

Vale mencionar que nas três áreas em estudo não há publicações que abordam de maneira específica a problemática de erros na administração de medicamentos.

Há um grande número de artigos publicados na área de farmácia, quando comparados ao número de publicações na área de enfermagem, embora, a administração de medicamentos seja considerada uma experiência diária nas atividades de enfermagem.

A partir da leitura dos artigos identificados, emergiu uma proposta de classificação que foi discutida e testada no grupo de pesquisa durante o processo de análise. Essa classificação abrange os seguintes aspectos (ordenados em ordem alfabética): assistência de enfermagem, ensino, medicamento, pesquisa e técnica.

O tema Assistência de enfermagem engloba assuntos sobre a problemática geral ou específica das características das atividades assistenciais das categorias que compõem a enfermagem, referente a administração de medicamentos. Como exemplo temos: "Assistência de Enfermagem na aplicação de sulfato de magnésio em pacientes portadoras de préeclâmpsia grave e eclâmpsia no H.C. de Porto Alegre".

Da categoria ensino, constaram artigos sobre programas educativos e de orientação de enfermagem na administração de medicamentos bem como artigos que abordaram métodos de inovação do ensino na área. Podemos citar: "Administração de injetáveis em farmácias: ação educativa do enfermeiro".

A categoria medicamentos, engloba artigos que tratam de questões diretamente ligadas à prescrição, distribuição, aquisição e dissolução de medicamentos. Como exemplo, temos: "Dissolução de medicamentos no Brasil: urgência na padronização".

$O$ item pesquisa refere-se a trabalhos voltados para o estudo da problemática geral da pesquisa relativa a administração de medicamentos ou para questões a ela relacionadas. Temos como exemplo: "A temática administração de medicamentos nas pesquisas em enfermagem".

Finalmente o tópico técnica refere-se a pesquisas sobre preparo e manuseio de medicamentos, possiveis regiões para administração dos mesmos e instrumental. Pode ser citado como exemplo: "O emprego de colheres domésticas para medir xaropes e suspensões medicamentosas".

Tabela 1 - Classificação dos artigos referentes à administração de medicamentos presentes nos periódicos revisados nas áreas de enfermagem, farmácia e hospital, no período de 1987 a 1997, segundo sua temática.

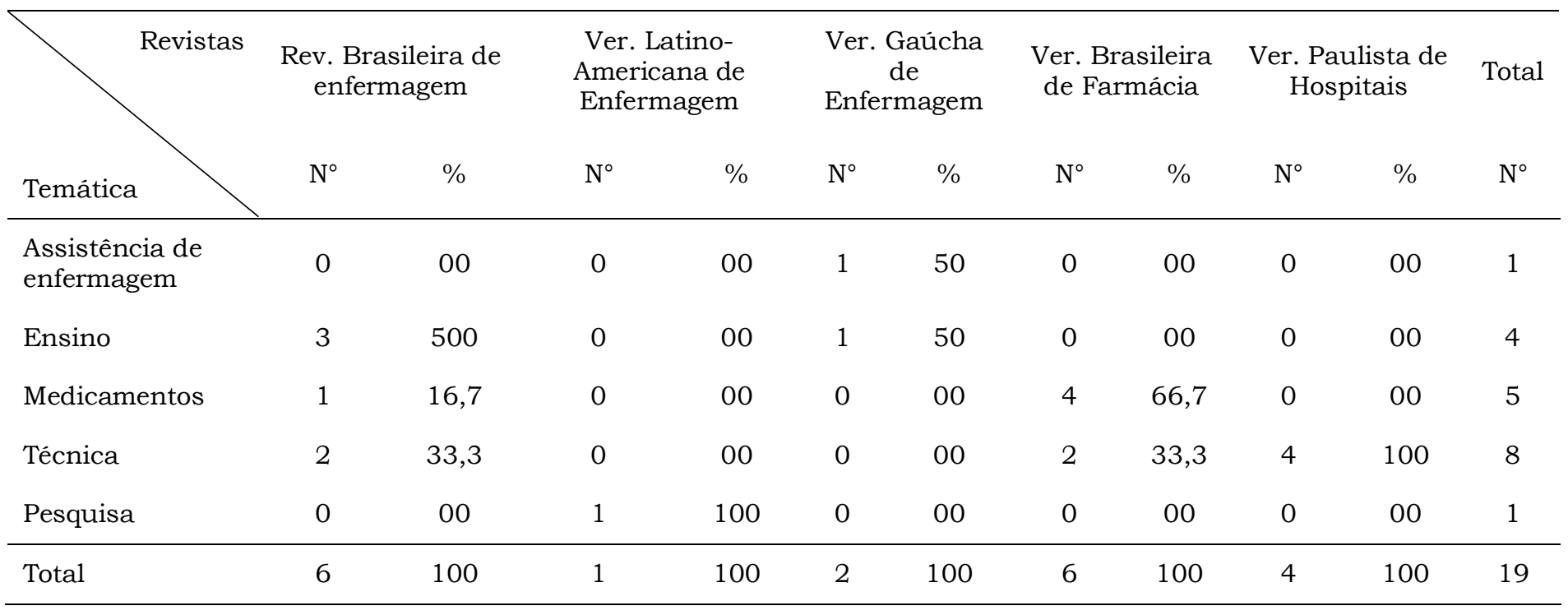


Conforme pode ser visualizado na Tabela 1 , encontramos um maior número de publicações $(42,1 \%)$ referentes aos aspectos técnicos da administração de medicamentos. Constatamos, portanto, que a problemática abarcada por esse item constitui-se na preocupação central dos pesquisadores e têm sido abordada nas três áreas em estudo, embora vários outros aspectos merecem atenção dos pesquisadores. Dentre eles, têm-se notado que erros na identificação dos pacientes no momento da administração de medicamentos têm ocorrido mais frequentemente.
Diante desse aspecto, decidimos também verificar a presença da identificação adequada no leito e no antebraço de pacientes internados em uma Instituição Hospitalar.

\section{IDENTIFICAÇÃO DO PACIENTE}

Foram verificados os trezentos e sessenta (360) leitos da instituição quanto a sua distribuição nas diferentes clínicas, ocupação e identificação dos mesmos bem como a identificação no antebraço do paciente.

Tabela 2 - Distribuição dos leitos, sua ocupação e identificação e o número de pacientes identificados nas diferentes Clínicas existentes na Instituição. Ribeirão Preto, 1998.

\begin{tabular}{lcccccccc}
\hline CLÍNICAS & \multicolumn{2}{c}{$\mathbf{N}^{\circ}$ DE LEITOS } & \multicolumn{2}{c}{$\begin{array}{l}\mathbf{N}^{\circ} \text { DE LEITOS } \\
\text { OCUPADOS }\end{array}$} & \multicolumn{3}{c}{$\begin{array}{c}\text { N DE LEITOS SEM } \\
\text { IDENTIFICAÇÃO }\end{array}$} & \multicolumn{3}{c}{$\begin{array}{c}\mathbf{N}^{\circ} \text { DE PACIENTES } \\
\text { COM PULSEIRAS }\end{array}$} \\
& $\mathrm{N}^{\circ}$ & $\%$ & $\mathrm{~N}^{\circ}$ & $\%$ & $\mathrm{~N}^{\circ}$ & $\%$ & $\mathrm{~N}^{\circ}$ & $\%$ \\
\hline PSIQUIATRICA & 14 & 3,9 & 14 & 100 & 02 & 14 & 00 & 00 \\
NEURO/DERMA & 44 & 12,2 & 33 & 75,0 & 1 & 3,0 & 14 & 42,4 \\
MÉDICA & 52 & 14,4 & 44 & 84,6 & 4 & 9,1 & 03 & 6,8 \\
PEDIATRICA & 30 & 8,3 & 29 & 96,7 & 2 & 6,9 & 03 & 10,3 \\
GINECOLÓGIA & 54 & 15,0 & 41 & 75,9 & 37 & 90,2 & 16 & 39,0 \\
CIRÚRGICA & 106 & 29,5 & 77 & 72,6 & 05 & 6,5 & 23 & 29,8 \\
ORTOPÉDICA & 38 & 10,6 & 16 & 42,0 & 14 & 87,5 & 02 & 12,5 \\
OFTALMICA & 22 & 6,1 & 15 & 68,2 & 00 & 00 & 03 & 20,0 \\
\hline TOTAL & 360 & 100 & 269 & 74,7 & 65 & 24,1 & 64 & 23,8 \\
\hline
\end{tabular}

Da tabela 2 evidencia-se a falta de utilização da pulseira de identificação do paciente na clínica psiquiátrica. Quando questionadas sobre o assunto, as enfermeiras desta Unidade justificaram o desuso da pulseira pelo pequeno número de pacientes (14), o que facilita o conhecimento dos nomes dos mesmos. Informam ainda que raramente há ocorrência de erro de medicação relacionado à paciente errado.

Observa-se ainda, que a clínica ginecológica é a que apresenta maior número de leitos sem identificação $(90,2 \%)$, entretanto ocupa o segundo lugar na utilização da pulseira de identificação no paciente. Vale ressaltar que nesta clínica funciona o sistema de alojamento conjunto que consiste na permanência do recém nascido em um berço próximo ao leito da mãe. Observou-se que a maioria das crianças possuíam a pulseira, o que não foi evidenciado em relação as mães. Há nesta clínica, vários pacientes em uso de quimioterápicos, podendo ocorrer até o óbito caso o paciente receba o medicamento errado.

Verificou-se que a clínica oftalmológica possui uma grande preocupação em identificar seus leitos $(100 \%)$, mas apenas $20 \%$ de seus pacientes possuem identificação no corpo.

$\mathrm{Na}$ clínica pediátrica, observou-se que apenas $10,34 \%$ dos pacientes faziam uso da pulseira de identificação. Vale lembrar que uma das alas dessa clínica é ocupada em sua maioria por crianças menores de um ano e que nem sempre estas estão acompanhadas pelas mães, dificultando o reconhecimento das crianças através da estratégia de perguntar-lhes o nome. $\mathrm{Na}$ ala ocupada pelas crianças maiores, há o constante 
uso de quimioterápicos, o que agrava a ocorrência de erros.

Em termos gerais, temos que dos 360 leitos da Instituição, 74,7\% destes estavam ocupados, $75,9 \%$ identificados e $23,8 \%$ dos pacientes possuíam a pulseira de identificação no antebraço, porcentagem esta maior do que a apresentada por PEPPER (1995), onde $11,4 \%$ dos pacientes da instituição estudada não possuíam a pulseira de identificação.

Tabela 3 - Utilização da pulseira de identificação no paciente nas diferentes clínicas da instituição. Ribeirão Preto, 1998.

\begin{tabular}{|c|c|c|c|c|c|c|c|c|c|c|c|c|}
\hline \multirow{2}{*}{ CLÍNICAS } & \multicolumn{2}{|c|}{$\begin{array}{l}\text { COLOCADA NO } \\
\text { MOMENTO DA } \\
\text { INTERNAÇÃOO }\end{array}$} & \multicolumn{2}{|c|}{$\begin{array}{l}\text { COLOCADA } \\
\text { APÓS } \\
\text { INTERNAÇÃO }\end{array}$} & \multicolumn{2}{|c|}{$\begin{array}{l}\text { VEIO DE OUTRA } \\
\text { INSTITUIÇÃO } \\
\text { (FOI TIRADA) }\end{array}$} & \multicolumn{2}{|c|}{$\begin{array}{l}\text { VEIO OUTRA } \\
\text { INSTITUIÇÃO } \\
\text { (FOI MANTIDA) }\end{array}$} & \multicolumn{2}{|c|}{$\begin{array}{l}\text { JÁ USARAM COMO } \\
\text { PREPARO } \\
\text { CIRÚRGICO }\end{array}$} & \multicolumn{2}{|c|}{$\begin{array}{l}\text { NUNCA } \\
\text { FIZERAM } \\
\text { USO }\end{array}$} \\
\hline & $\mathrm{N}^{\circ}$ & $\%$ & $\mathrm{~N}^{\circ}$ & $\%$ & $\mathrm{~N}^{\circ}$ & $\%$ & $\mathrm{~N}^{\circ}$ & $\%$ & $\mathrm{~N}^{\circ}$ & $\%$ & $\mathrm{~N}^{\circ}$ & $\%$ \\
\hline PSIQUIÁTRICA & 00 & 00 & 00 & 00 & 11 & 19,6 & 00 & 00 & 00 & 00 & 03 & 5,0 \\
\hline NEURO/DERMA & 02 & 18,2 & 07 & 21,2 & 06 & 10,7 & 05 & 25,0 & 06 & 6,7 & 07 & 12,2 \\
\hline MÉDICA & 00 & 00 & 01 & 3,0 & 10 & 17,9 & 02 & 10,0 & 19 & 21,1 & 12 & 20,3 \\
\hline PEDIÁTRICA & 02 & 18,2 & 00 & 00 & 07 & 12,5 & 01 & 5,0 & 09 & 10,0 & 10 & 17,0 \\
\hline GINECOLÓGICA & 04 & 36,3 & 08 & 24,2 & 03 & 5,4 & 04 & 20,0 & 16 & 17,8 & 06 & 10,1 \\
\hline CIRÚRGICA & 03 & 27,3 & 13 & 39,4 & 09 & 16,0 & 07 & 35,0 & 28 & 31,1 & 17 & 28,8 \\
\hline ORTOPÉDICA & 00 & 00 & 02 & 6,1 & 07 & 12,5 & 00 & 00 & 05 & 5,5 & 02 & 3,3 \\
\hline OFTALMOLÓGICA & 0 & 00 & 02 & 6,1 & 03 & 5,4 & 01 & 5,0 & 07 & 7,8 & 02 & 3,3 \\
\hline TOTAL & 11 & 4,1 & 33 & 12,3 & 56 & 20,8 & 20 & 7,4 & 90 & 33,4 & 59 & 22,0 \\
\hline
\end{tabular}

A Tabela 3 mostra que a clínica ginecológica é a que mais identifica o paciente no momento da internação, embora possa ser observado que, nesta instituição, apenas $4,1 \%$ dos pacientes têm sido identificados nesta ocasião.

A clínica cirúrgica tem um maior número de pacientes identificados após a internação, bem como de pacientes que já fizeram uso da pulseira como preparo cirúrgico. Vale ressaltar que dos pacientes identificados após a internação, a maioria ocorreu no dia anterior a cirurgia e teve a pulseira mantida posteriormente. O item "pacientes que já usaram a pulseira como preparo cirúrgico", engloba pacientes que já usaram a identificação como preparo cirúrgico em internações anteriores e pacientes que embora fizeram uso na atual internação, tiveram a pulseira retirada após a cirurgia.

Observa-se que a clínica psiquiátrica não identifica seus pacientes através da pulseira e retira esta do antebraço do paciente por ocasião de sua internação.

Dos 269 pacientes entrevistados, verifica-se que $33,4 \%$ tiveram a pulseira colocada no dia anterior à cirurgia e apenas $4,1 \%$ no momento da internação. Este fato se agrava ao verificarmos que
$20,8 \%$ dos pacientes vieram transferidos de outras instituições, usando a pulseira de identificação, e que esta foi tirada por ocasião da internação na instituição em estudo. Quando questionados, a maioria dos pacientes informou não saber o motivo pelo qual a pulseira foi tirada. Observa-se ainda que $22 \%$ dos pacientes nunca fizeram uso da pulseira de identificação. Destes últimos, alguns informam ser esta a primeira internação e outros referem breves internações anteriores, sem ato cirúrgico.

Um levantamento recente feito nesta Instituição revelou que a intercorrência mais freqüente ocorrida na administração de medicamentos $(33,9 \%)$ consiste no paciente errado. Pode-se supor, portanto, que a falta de identificação tanto no leito, quanto do paciente, pode ser um dos fatores que esteja levando à ocorrência de erros na administração de medicamentos.

\section{CONSIDERAÇÕES FINAIS}

Tendo em vista que a administração de medicamentos constitui uma importante atividade da equipe de Enfermagem, e.que uma das intercorrências 
mais freqüentes nesta atividade consiste no paciente errado, consideramos importante verificar se os pacientes internados em uma Instituição Hospitalar apresentavam identificação adequada no leito e no antebraço.

De acordo com os resultados obtidos, pudemos verificar que dos 360 leitos da Instituição, 74,7\% destes estavam ocupados, $76 \%$ destes identificados e $23,8 \%$ dos pacientes possuíam a pulseira de identificação no antebraço.

Observamos que $33,4 \%$ dos pacientes entrevistados receberam a pulseira no antebraço no dia anterior a cirurgia e apenas $4,1 \%$ no momento da internação. Este fato agrava-se ao verificarmos que um grande número de pacientes têm sido transferidos de outras Instituições usando a pulseira de identificação e que esta tem sido retirada (20,8\%) após internação na Instituição em estudo.

Quanto ao levantamento bibliográfico nas áreas de enfermagem, farmácia e hospitalar, observou-se uma pequena quantidade de publicações sobre a temática estudada: dezenove artigos, sendo nove destes publicados na área de enfermagem. Vale ressaltar que não foram encontrados nestas áreas, artigos que abordassem de maneira específica a problemática de erros na administração de medicamentos.

No que concerne a classificação dos artigos, verificamos um maior número de publicações $(42,1 \%)$ voltadas para os aspectos técnicos da administração de medicamentos nas três áreas em estudo, revelando ser esta a preocupação central dos pesquisadores.

Os resultados evidenciam a pouca importância da identificação do paciente e do leito por parte da instituição e a necessidade de assegurar que todos os pacientes tenham sua identificação durante toda a internação nas instituições de saúde.

PEPPER (1995) afirma que um individuo raramente é a única causa de um erro de medicação e que os erros deveriam ser considerados como falhas de sistemas, ao invés de falhas do individuo. Para este autor, falhas no cumprimento da política institucional é um grande fator de erros na administração de medicamentos. A participação da enfermagem na solução de problemas é o melhor caminho para derivar estratégias que serão efetivas e práticas.

É preciso então, além da conscientização do problema, que exista a vontade política daqueles que estão na direção dos serviços, de fazer valer as normas e recomendações do sistema em que se inserem, educando os profissionais envolvidos quanto a importância da adoção de medidas que visem minimizar a ocorrência de erros, entre eles a medicação administrada à pacientes errados por falta de identificação do leito e no antebraço do paciente e questionaménto do nome do paciente.

\section{REFERÊNCIAS BIBLIOGRÁFICAS}

BARALDI, S.; OLIVEIRA, S.L.; OLIVEIRA, M.A. A utilização da região ventro-glútea para aplicação da vacina dupla-adulto: opinião da clientela. Rev. Bras. Enf., v.47, n.3, p.314-24, 1994.

BOOTH, B. Managemente of drug errors. Nurs.Times., v.90, n. 15 , p. $30-31,1994$.

BRAllACH, M.L.; BERTUZZI, H.J.; BOLYHOS, I. Cuidados na administração de alguns medicamentos injetáveis. Rev. Paul. Hosp., v.35, n. 7/8/9, p.112-5, 1987.

CARESTIATO, J.C.; FERREIRA, L.G. Dose unitária: relação custo x beneficio de sua implantação nos hospitais públicos brasileiros como política de governo. Rev. Bras. Farm., v.77,n.3, p.103-12, 1996.

CASSIANI, S.H.B.; BUENO,E. Erros na administração de medicamentos segundo um grupo de enfermeiros em um hospital universitário do interior paulista: fatores de risco e medidas empregadas. In: COLÓQUIO PANAMERICANO DE ENFERMAGEM, 6, Ribeirão Preto, 1998. Livro de Resumos. Ribeirão Preto, Centro Colaborador da OMS para o Desenvolvimento da Pesquisa em Enfermagem, 1998. p.14.

CASTRO, C.G.S.O.; RIBEIRO, D.C.S.; PITTA, A.C.M. Perfil de prescrição de soluções nutritivas parenterais no IFF/FIO CRUZ: estudo em três enfermarias pediátricas, 193/94. Rev. Bras. Farm., v.75, n.4, p.86-90, 1994.

CASTRO, C.G.S.O.; RIBEIRO, D.C.S. Protocolo para aquisição de medicamentos em instituições públicas de saúde. Rev. Bras. Farm., v.77, n.3, p.85-7, 1996.

COBB, M.D. Evaluating medication errors. J. Nurs. Adm., v.16, n.4, p.41-4, 1986.

CZAPSKI, J. et al.. Normas para o manuseio em serviços de saúde de soluções de grande volume para uso parenteral. Rev. Paul. Hosp., v.36, n.10/11/12, p.163-66, 1988.

DUARTE, M.J.R.S. Influência da indústria farmacêutica na política de medicamentos, segundo os profissionais de saúde. Rev. Bras. Enf., v.44, n.2, p.10-20, 1991.

DUARTE, N.M.N. et al.. Assistência de enfermagem na aplicação de sulfato de magnésio em pacientes portadoras de pré-eclâmpsia grave e eclâmpsia no Hospital de Clínicas de Porto Alegre HCPA. Rev. Gaúcha Enf., v.8, n.1, p.1-16, 1987.

FÁTIMA, A.P. A temática administração de medicamentos nas pesquisas em enfermagem. Rev. Lat-Amer. Enf., v.1, p.129, 1993, n. especial.

LISBOA, S.M.; GRABE, A. O emprego de colheres domésticas para medir xaropes e suspensões medicamentosas. Rev. Bras.Farm., v. 73, n.4, p.9'7-9, 1992.

MAEDA, S.T.; CAMPEDELLI, M.C. Escalpe e equipo de soro: opinião de enfermeiras quanto aos requisitos essenciais de qualidade. Rev. Bras.Enf., v.44, n.3, p.124-34, 1991.

MENDES, I.A.C. et al.. A administração de injetáveis em farmácias: ação educativo do enfermeiro. Rev. Bras. Enf., v.41, n.2, p.93-6, 1988. 
MILANEZ, H.M.; ALMERÃO, E. O papel da enfermeira na orientação e administração de drogas que levam à imunossupressão em pacientes submetidos à transplante cardiaco. Rev.Gaúcha Enf., v.8, n.2, p.220-26, 1987.

MIQUELIN, J.D.L.; CASSIANI, S.H.B.; BUENO, E. Administração de medicamentos: revisão de relatórios de intercorrências. In: COLÓQUIO PANAMERICANO DE ENFERMAGEM, 6, Ribeirão Preto, 1998. Livro de Resumos. Ribeirão Preto, Centro Colaborador da OMS para o Desenvolvimento da Pesquisa em Enfermagem, 1998. p.217.

MIYADAHIRA, A.M.K.; KOIZUMI, M.S. Medida das capacidades psicomotoras envolvidas na técnica da injeção intramuscular. Rev. Bras. Enf., v.45, n.4, p.166-277, 1992.

NASCIMENTO, E.M.F.; CANÇADO, M.L.B. O uso de um sistema artesanalmente construído para o ensino prático da punção e infusão intravenosa. Rev. Bras. Enf., v.44, n.4, p.18-21, 1991.
PEPPER, G.A. Errors in drug administration by nurses. Am. J. Health System Pharm., v.52, p.390-5, 1995.

ROSA, V.H.C.T.; MARIN, M.L.M.; BARBOSA, A.O. Requisitos necessários para o preparo asséptico das soluções de Nutrição Parenteral Prolongada (NPP). Rev. Bras.Farm.., v.73, n.4, p.93-6, 1992

ROSA, V.H.C.T.; PIVELLO, V.L. Antibióticos e antineoplásicos de uso injetável: cuidados nos procedimentos de manuseio: fator de segurança e economia. Rev.Paul. Hosp., v.35, n.4/5/6, p.71-8, 1987.

SANTORO, M.I.R.M. Dissolução de medicamentos no Brasil: urgência na padronização. Rev. Bras. Farm., v.72, n.1, p.11-2, 1991.

SCAIN, S.F. Reutilização de seringa descartável para aplicação de insulina. Rev.Paul.Hosp., v.36, n.1/2/3, p. 18-23, 1988. 